Article

\title{
Propagation, Inactivation, and Safety Testing of SARS-CoV-2
}

\author{
Alexander S. Jureka ${ }^{+} \mathbb{D}$, Jesus A. Silvas ${ }^{+} \mathbb{D}$ and Christopher F. Basler * \\ Center for Microbial Pathogenesis, Institute for Biomedical Sciences, Georgia State University, \\ Atlanta, GA 303030, USA; ajureka@gsu.edu (A.S.J.); jsilvas@gsu.edu (J.A.S.) \\ * Correspondence: cbasler@gsu.edu; Tel.: +1-404-413-3651 \\ t Those authors contributed equally to this manuscript.
}

Received: 13 May 2020; Accepted: 4 June 2020; Published: 6 June 2020

\begin{abstract}
In late 2019, a novel coronavirus, severe acute respiratory syndrome coronavirus 2 (SARS-CoV-2) emerged in Wuhan, the capital of the Chinese province Hubei. Since then, SARS-CoV-2 has been responsible for a worldwide pandemic resulting in over 4 million infections and over 250,000 deaths. The pandemic has instigated widespread research related to SARS-CoV-2 and the disease that it causes, COVID-19. Research into this new virus will be facilitated by the availability of clearly described and effective procedures that enable the propagation and quantification of infectious virus. As work with the virus is recommended to be performed at biosafety level 3 , validated methods to effectively inactivate the virus to enable the safe study of RNA, DNA, and protein from infected cells are also needed. Here, we report methods used to grow SARS-CoV-2 in multiple cell lines and to measure virus infectivity by plaque assay using either agarose or microcrystalline cellulose as an overlay as well as a SARS-CoV-2 specific focus forming assay. We also demonstrate effective inactivation by TRIzol, 10\% neutral buffered formalin, beta propiolactone, and heat.
\end{abstract}

Keywords: SARS-CoV-2; coronavirus; virus; virology; plaque assay; inactivation

\section{Introduction}

The novel coronavirus SARS-CoV-2, the causative agent of Coronavirus disease 2019 (COVID-19), belongs to the betacoronavirus genus, which also includes the highly pathogenic SARS CoV and MERS CoV. SARS-CoV-2, a close relative of bat betacoronaviruses emerged at the end of 2019 in Wuhan, China, and has caused a pandemic [1]. The pandemic and the public response have greatly impacted public health and caused profound social and economic disruption. As of May 11, 2020, SARS-CoV-2 had caused more than four million infections and more than 250,000 deaths worldwide [2]. The emergence of this new virus has prompted urgent worldwide efforts to develop diagnostics, vaccines, and antivirals, to define the natural history of human infection; and to better characterize the virus.

The expanded interest in studying SARS-CoV-2 to address the current pandemic requires that many laboratories acquire the capacity to work with the virus. However, despite the rapidly growing body of literature either deposited on preprint servers or in peer-reviewed scientific journals, there remains a lack of information regarding standardized protocols for work with the virus. Among these needs are the means to grow and quantify infectious virus. Additionally, the recommendation that experiments involving the propagation of SARs-CoV-2 be performed at biosafety level 3 (BSL3) necessitates the development of methods to safely inactivate the virus and validate inactivation methods to allow an array of studies to be performed at lower biocontainment levels [3-6]. Examples include the isolation of RNA from virus and virus-infected cells to characterize viral genome sequences, monitor viral gene 
expression and genome replication, and to characterize host responses to infection. The removal of intact, virus-infected cells is critical for studies involving microscopy. Whole inactivated virus and viral proteins are needed for the development of inactivated whole-virus vaccine preparations, and also as a source of antigen for immunoassays.

To help address these needs and to facilitate SARS-CoV-2 research efforts, we describe here methods for the propagation of SARS-CoV-2 in multiple cell lines. We have also determined a more efficient method for quantifying virus by plaque assay and have developed a SARS-CoV-2-specific focus forming assay which can enhance throughput of assays requiring quantification of viral titers. Additionally, we describe validation of methods for the inactivation of SARS-CoV-2 through the use of TRIzol, 10\% neutral buffered formalin, beta-propiolactone, and heat. Taken together, the data presented here will serve to provide researchers with a helpful basis of information to aid in their work on SARS-CoV-2.

\section{Materials and Methods}

\subsection{Cells and Virus}

Vero E6 (ATCC\# CRL-1586), Calu-3 (ATCC\# HTB-55), Caco-2 (ATCC\# HTB-37), Huh7, A549 (ATCC\# CCL-185), and 293T cells were maintained in DMEM (Corning) supplemented with 10\% heat inactivated fetal bovine serum (FBS; GIBCO). Cells were kept in a $37^{\circ} \mathrm{C}, 5 \% \mathrm{CO}_{2}$ incubator without antibiotics or antimycotics. SARS-CoV-2, strain USA_WA1/2020, was obtained from the World Reference Collection for Emerging Viruses and Arboviruses at the University of Texas Medical Branch-Galveston.

\subsection{Virus Propagation}

A lyophilized ampule of SARS-CoV-2 was initially resuspended in DMEM supplemented with $2 \%$ FBS. VeroE6 cells were inoculated in duplicate with a dilution of 1:100 with an adsorption period of $1 \mathrm{~h}$ at $37^{\circ} \mathrm{C}$ and shaking every $15 \mathrm{~min}$. Cells were observed for cytopathic effect (CPE) every $24 \mathrm{~h}$. Stock SARS-CoV-2 virus was harvested at $72 \mathrm{~h}$ post infection (h.p.i) and supernatants were collected, clarified, aliquoted, and stored at $-80^{\circ} \mathrm{C}$. For replication kinetic experiments, cells were seeded into 24-well plates at confluency in DMEM supplemented in 10\% FBS. The next day, cells were inoculated with SARS-CoV-2 at a multiplicity of infection (MOI) of 0.01 in DMEM supplemented with $2 \%$ FBS. Supernatants were harvested at the indicated timepoints and stored at $-80^{\circ} \mathrm{C}$ until analysis.

\subsection{SARS-CoV-2 Plaque Assay}

Vero E6 cells were seeded into 6, 12, or 24-well plates $24 \mathrm{~h}$ before infection. Ten-fold serial dilutions of SARS-CoV-2 samples were added, adsorbed for $1 \mathrm{~h}$ at $37{ }^{\circ} \mathrm{C}$ with shaking at 15 -min intervals. After the absorption period, $2 \mathrm{~mL}$ of $0.3 \%$ agarose in DMEM supplemented with $2 \%$ FBS or $3 \mathrm{~mL}$ of 0.6 or 1.2 percent microcrystalline cellulose (MCC; Sigma 435244, St. Louis, MO, USA) in serum-free DMEM was added. For MCC plaque assays, virus inoculum was not removed. To stain plaque assays performed with an agarose overlay, 10\% neutral buffered formalin (NBF) was added on top of the agarose and incubated for one hour at room temperature. The agarose plug was then removed with a pipette tip, and the fixed monolayer was stained with $0.4 \%$ crystal violet in $20 \%$ methanol. For plaque assays performed with MCC overlay, the MCC was aspirated out, 10\% NBF added for one hour at room temp and then removed. Monolayers were then washed with water and stained with $0.4 \%$ crystal violet. Plaques were quantified and recorded as PFU/mL.

\subsection{Focus Forming Assay}

Vero E6 cells were plated into 96-well plates at confluency (75,000 cells/well) in DMEM supplemented with $10 \%$ heat-inactivated fetal bovine serum (Gibco). Prior to infection, virus stocks were thawed and serially diluted to obtain dilutions in the range of $10^{-2}$ to $10^{-9}$. Growth media was removed from the Vero E6 cells and $50 \mu \mathrm{L}$ of virus dilutions was plated. Virus was adsorbed for $1 \mathrm{~h}$ at 
$37^{\circ} \mathrm{C} / 5 \% \mathrm{CO}_{2}$. After adsorption, $50 \mu \mathrm{L}$ of $2.4 \%$ MCC overlay supplemented with DMEM powdered media to a concentration of $1 \mathrm{X}$ (Gibco) was added to each well of the 96-well plate to achieve a final MCC overlay concentration of $1.2 \%$. Plates were then incubated at $37{ }^{\circ} \mathrm{C} / 5 \% \mathrm{CO}_{2}$ for $24 \mathrm{~h}$. The MCC overlay was gently removed and cells were fixed with $10 \% \mathrm{NBF}$ for $1 \mathrm{~h}$ at room-temperature. After removal of $\mathrm{NBF}$, monolayers were washed with ultrapure water and $100 \%$ methanol $/ 0.3 \% \mathrm{H}_{2} \mathrm{O}_{2}$ was added to permeabilize cells and quench endogenous peroxidase activity. Monolayers were then blocked for $1 \mathrm{~h}$ in PBS with 5\% non-fat dry milk (NFDM). After blocking, monolayers were incubated with SARS-CoV N primary antibody (Novus Biologicals, Centennial, CO, USA; NB100-56576-1:2000) for $1 \mathrm{~h}$ at RT in PBS/5\% NFDM. Monolayers were washed with PBS and incubated with an HRP-Conjugated secondary antibody for $1 \mathrm{~h}$ at RT in PBS/5\% NFDM. Secondary was removed, monolayers were washed with PBS, and then developed using TrueBlue substrate (KPL) for $30 \mathrm{~min}$. Plates were imaged on a Bio-Rad Chemidoc utilizing a phosphorscreen and foci were quantified.

\subsection{TRIzol ${ }^{\circledR}$ Treatment}

To validate the effectiveness of Trizol ${ }^{\circledR}$ Reagent in the inactivation of SARS-CoV-2, stock virus was separated into the following validation test groups: non-infected control (sterile media), Trizol treated non-infected control (sterile culture media + Trizol), duplicate SARS-CoV-2 positive controls (SARS-CoV-2; $1 \times 10^{6} \mathrm{pfu}$ ), and triplicate SARS-CoV-2 Trizol treated samples (SARS-CoV-2; $1 \times 10^{6} \mathrm{pfu}+$ Trizol). Trizol was added to a final concentration of $10 \%$. All test groups were then incubated at room temperature for $10 \mathrm{~min}$. Samples were then diluted in $25 \mathrm{~mL}$ media and plated onto VeroE6 cells in a $15 \mathrm{~cm}$ tissue culture dish, incubated at $37^{\circ} \mathrm{C}$ and $5 \% \mathrm{CO}_{2}$, and monitored daily for CPE. At $48 \mathrm{~h}$ post infection, media was removed and the plates fixed with $10 \%$ NBF. To determine integrity of the cell monolayer, plates were stained with crystal violet.

\subsection{Formalin Treatment}

To validate inactivation of SARS-CoV-2 with formalin, 21 wells of a 24-well plate seeded to confluency with VeroE6 cells were infected with SARS-CoV-2 at an MOI of 0.1. Three wells served as mock-infected control. At $24 \mathrm{~h}$ post infection, media was removed from wells and treated as follows, using 3 wells per condition: uninfected control, SARS-CoV-2 infected control, SARS-CoV-2 + 2\% formaldehyde, SARS-CoV-2 + 1\% formaldehyde, SARS-CoV-2 + 0.5\% formaldehyde, SARS-CoV-2 $+0.1 \%$ formaldehyde, and SARS-CoV-2 + 0.05\% formaldehyde. Formaldehyde treatment was carried out at room temperature for $1 \mathrm{~h}$. The cells were then washed with fresh media, scraped off the plate, and overlaid onto uninfected Vero E6 cells. Samples were then incubated at $37{ }^{\circ} \mathrm{C}, 5 \% \mathrm{CO}_{2}$ and monitored daily for CPE. At $72 \mathrm{~h}$ post infection, media was removed and the plates fixed with $10 \% \mathrm{NBF}$. To determine integrity of the cell monolayer, plates were stained with crystal violet.

\subsection{Beta-Propiolactone Treatment}

To test treatment of viral particles with beta-propiolactone as means of inactivation, stock SARS-CoV-2 virus was separated into the following validation groups: non-infected control, $4{ }^{\circ} \mathrm{C}$ control $\left(1 \times 10^{6} \mathrm{pfu}\right.$ per replicate $), 0.5 \%$ beta-propiolactone $\left(1 \times 10^{6}\right.$ pfu per replicate $)$, $0.1 \%$ beta-propiolactone $\left(1 \times 10^{6} \mathrm{pfu}\right.$ per replicate), and $0.05 \%$ beta-propiolactone $\left(1 \times 10^{6} \mathrm{pfu}\right.$ per replicate). After incubation at $4{ }^{\circ} \mathrm{C}$ for $16 \mathrm{~h}$, samples were transferred to $37^{\circ} \mathrm{C}$ for two (2) hours to hydrolyze all residual beta-propiolactone. This step ensures complete hydrolysis of beta-propiolactone to prevent cytotoxicity to mammalian cells [7]. After hydrolysis, samples were inoculated onto Vero E6 cells, and incubated at $37^{\circ} \mathrm{C}, 5 \% \mathrm{CO}_{2}$ incubator and monitored daily for CPE. At $72 \mathrm{~h}$ post infection, media was removed and plates were fixed with $10 \%$ NBF. To determine integrity of the cell monolayer, plates were stained with crystal violet. 


\subsection{Heat Inactivation}

To validate heat treatment as a method to inactivate SARS-CoV-2, SARS-CoV-2 virus was separated into the following validation groups: non-infected control, room temperature control $\left(1 \times 10^{6}\right.$ pfu per replicate), $100{ }^{\circ} \mathrm{C}$ for $5 \mathrm{~min}\left(1 \times 10^{6} \mathrm{pfu}\right.$ per replicate), $100{ }^{\circ} \mathrm{C}$ for $10 \mathrm{~min}\left(1 \times 10^{6} \mathrm{pfu}\right.$ per replicate $)$, and $100{ }^{\circ} \mathrm{C}$ for $15 \mathrm{~min}\left(1 \times 10^{6} \mathrm{pfu}\right.$ per replicate). microcentrifuge polypropylene tubes $(1.5 \mathrm{~mL})$ containing the virus (500 $\mu \mathrm{L}$ total volume) were exposed to direct heat in a heat block (Fisher Scientific, Hampton, NH, USA). After heating, all samples were left to cool to room temperature and centrifuged to collect condensation within the tube. Each sample was then inoculated in 5-fold serial dilutions onto VeroE6 cells. Samples were then incubated at $37^{\circ} \mathrm{C}, 5 \% \mathrm{CO}_{2}$. At $72 \mathrm{~h}$ post infection, supernatants were harvested, and infectious virus was quantified by plaque assay on Vero E6 cells.

\subsection{Electron Microscopy}

Resuspended purified beta-propiolactone treated SARS-CoV-2 virus preps were adsorbed onto 300 mesh formvar-carbon coated nickel grids for $10 \mathrm{~min}$, washed with $0.2 \mu \mathrm{M}$ filtered ultrapure water, and negative-stained with UranyLess stain for $15 \mathrm{~s}$. Grids were then washed with $0.2 \mu \mathrm{M}$ ultrapure water, allowed to dry, and imaged on a LEO 960 TEM at $80 \mathrm{kV}$.

\subsection{Western Blotting}

Resuspended purified beta-propiolactone treated SARS-CoV-2 virus preps were separated by SDS-PAGE (Bio-Rad TGX Mini, Bio-Rad, Hercules, CA, USA) and transferred to 0.2 $\mu$ M PVDF membrane according to the manufacturer's protocols (Bio-Rad Tansblot Turbo; Bio-Rad, Hercules, CA, USA). After blocking in 5\% non-fat dry milk in TBST (10 mM Tris, $150 \mathrm{mM} \mathrm{NaCl}$, 0.5\% Tween-20, pH8) for one hour, membranes were incubated overnight at $4{ }^{\circ} \mathrm{C}$ with antibodies targeting SARS-CoV N (Novus Biologicals, Centennial, CO, USA; NB100-56576) or SARS-CoV S (Sino Biological, Wayne, PA, USA; 40150-T62). Membranes were washed in TBST and incubated with an HRP-conjugated rabbit secondary antibody (Cell signaling, Danvers, MA, USA; 7074) for $1 \mathrm{Hr}$ at room temperature. Membranes were then washed, developed with ECL, and imaged on a Bio-Rad Chemidoc imaging system.

\section{Results}

\subsection{Propagation and Quantification of SARS-CoV-2 in Cell Culture}

Despite the number of recent reports in which SARs-CoV-2 has been propagated and quantified, there still remains a lack of general information regarding the temporal cytopathology of SARS-CoV-2 in cell culture. Here, we set out to determine the most appropriate times post-infection to harvest SARS-CoV-2 infected cultures for generation of stock virus, and the quantification thereof. To generate stock virus, Vero E6 cells were infected with SARS-CoV-2 at an MOI of 0.001 and monitored daily by light microscopy for the appearance of CPE. We determined that the cytopathic effect (CPE) caused by SARS-CoV-2 in Vero E6 cells is most apparent at $48 \mathrm{~h}$ post infection (Figure 1A). However, despite $\mathrm{CPE}$ being nearly complete at $48 \mathrm{~h}$ post-infection in the absence of an overlay, we determined that for the quantification of SARS-CoV-2 using traditional plaque assays with a $0.4 \%$ agarose overlay $72 \mathrm{~h}$ post-infection results in clearer, more easily quantifiable plaques (Figure 1B). 
A.

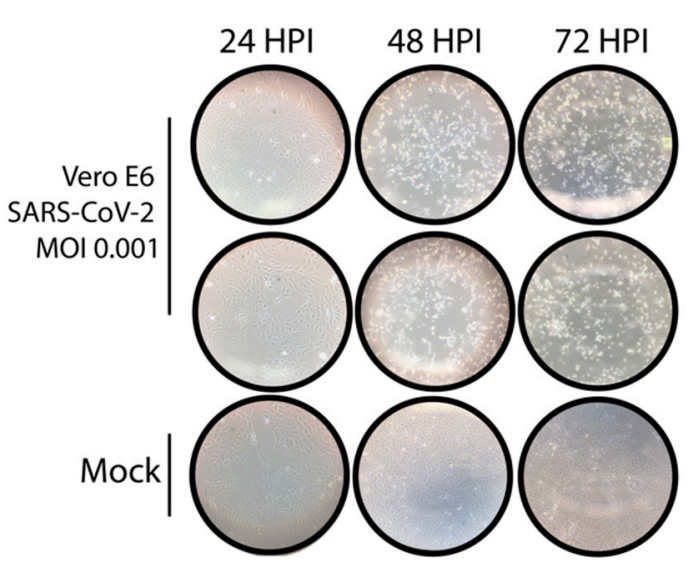

B.

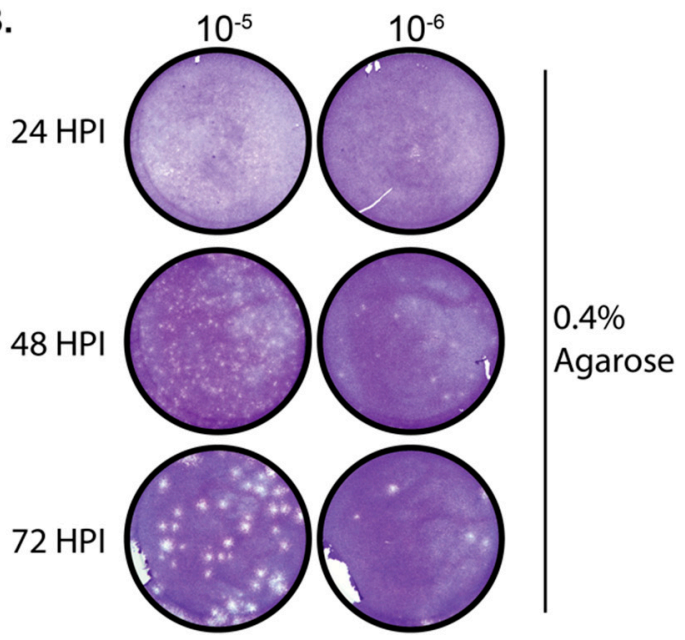

C.

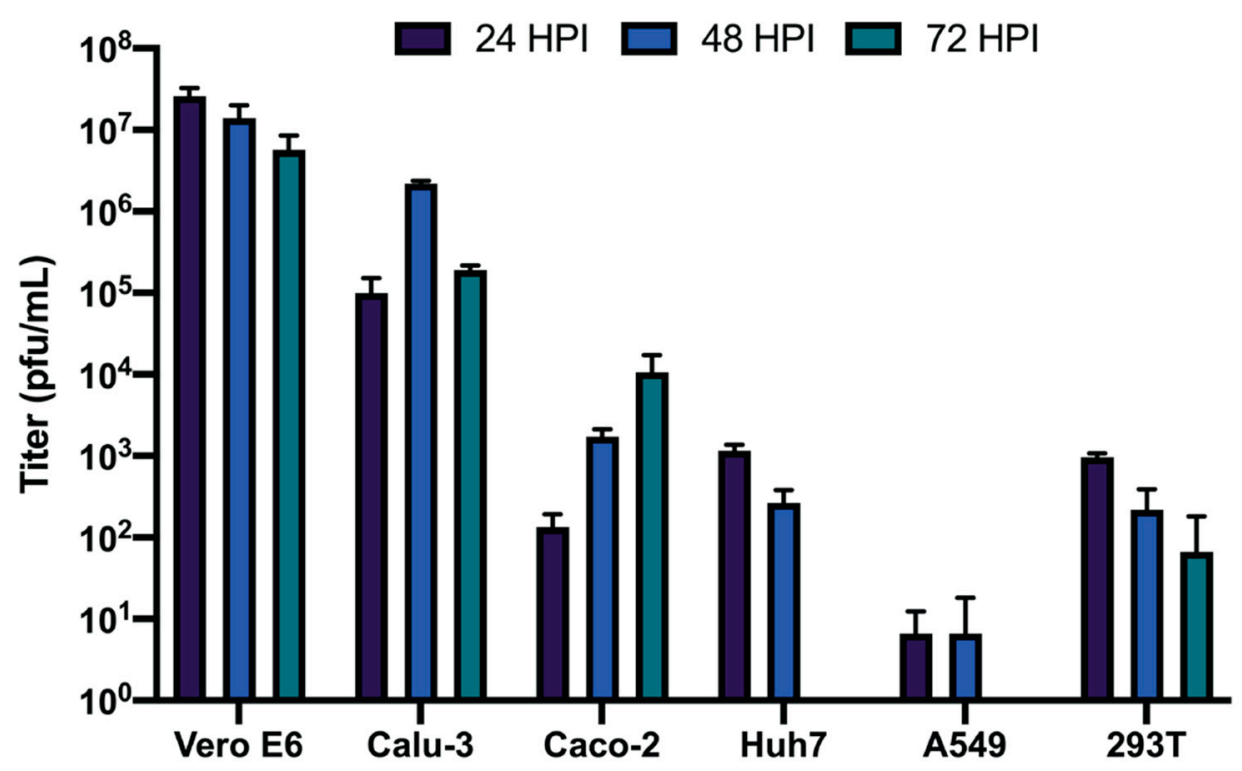

Figure 1. Propagation and quantification of SARS-CoV-2 in cell culture. (A) Vero E6 cells were infected with SARS-CoV-2 at an MOI of 0.001 and monitored daily by microscopy for the presence of cytopathic effect (CPE). (B) Vero E6 cells were infected with serially diluted SARS-CoV-2 stock virus and overlaid with $0.4 \%$ agarose in DMEM supplemented with $2 \%$ FBS. Plaque assays were harvested at 24, 48, and $72 \mathrm{~h}$ post-infection, fixed, and stained with crystal violet to visualize plaques. (C) Vero E6, Calu-3, Caco-2, Huh7, A549, and 293T cells were infected with SARS-CoV-2 at an MOI of 0.01. Supernatants from 24, 48, and 72-h timepoints were quantified by plaque assay. Data are representative of the mean and SEM of 3 replicates.

In addition, we wanted to determine the kinetics of SARS-CoV-2 in several commonly used cell lines to determine suitable cell culture systems for studying SARS-CoV-2 biology. Vero E6, Calu-3, Caco-2, Huh7, A549, and 293T cells were infected with SARS-CoV-2 at an MOI of 0.01 and supernatants were harvested at 24,48 , and $72 \mathrm{~h}$ post-infection and quantified by traditional plaque assays. As shown in Figure 1C, SARS-CoV-2 replicated to high titers in Vero E6 and Calu3 cells at all times post-infection. Caco-2 cells clearly support SARS-CoV-2 replication yet seem to propagate the virus more slowly than Vero E6 or Calu-3 cells. Only modest replication was observed in Huh7 and 293T cells, and A549 cells did not support SARS-CoV-2 growth at any time post-infection. These results suggest that the cellular tropism for SARS-CoV-2 is fairly restricted; however, overexpression of the SARS-CoV-2 receptor ACE2 
or the protease responsible for cleaving SARS-CoV-2 spike protein can aid infection in non-permissive cell lines $[8,9]$.

\subsection{Microcrystalline Cellulose is a Suitable Substitute for Agarose for SARS-CoV-2 Plaque Assays}

Traditionally, viral plaque assays are performed utilizing low concentrations of agarose as an overlay medium. While agarose generally performs well as an overlay medium for numerous different viruses, the requirement of having to remove agarose plug from within multi-well cell culture dishes can prove laborious. This is especially the case when plaque assays are performed in 24-well plates. Recently, a novel low-viscosity overlay medium for viral plaque assays has been described [10]. This assay replaces traditional solid (agarose) and semi-solid (methylcellulose) media with a microcrystalline cellulose (MCC) suspension as the overlay media. To determine whether the MCC approach is suitable for quantifying SARS-CoV-2 by plaque assay, we overlaid SARS-CoV-2 infected Vero E6 cells with two concentrations of MCC (0.6\% and 1.2\%) in 6, 12, and 24-well formats. Our results indicate that MCC performs exceptionally well as an overlay medium for SARS-CoV-2 plaque assays as uniform and countable plaques are readily apparent $72 \mathrm{~h}$ post-infection, even in the 24-well plate format (Figure 2A,B). We have also determined that either of the two concentrations of MCC tested yielded plaques of comparable size. Based on this we would recommend the use of $0.6 \%$ MCC overlay media (Figure 2A) for SARS-CoV-2 plaque assays as it produces uniform and easily identifiable plaques for quantification. While it is possible to identify plaques at $48 \mathrm{~h}$ post-infection with $0.6 \%$ MCC overlay, similar to the $0.4 \%$ agarose overlay (Figure 1B), the plaques are small and difficult to accurately quantify until $72 \mathrm{~h}$ post-infection. Taken together these data demonstrate that using MCC as an overlay media is an effective and far more efficient method than the use of traditional agarose overlays.

A.

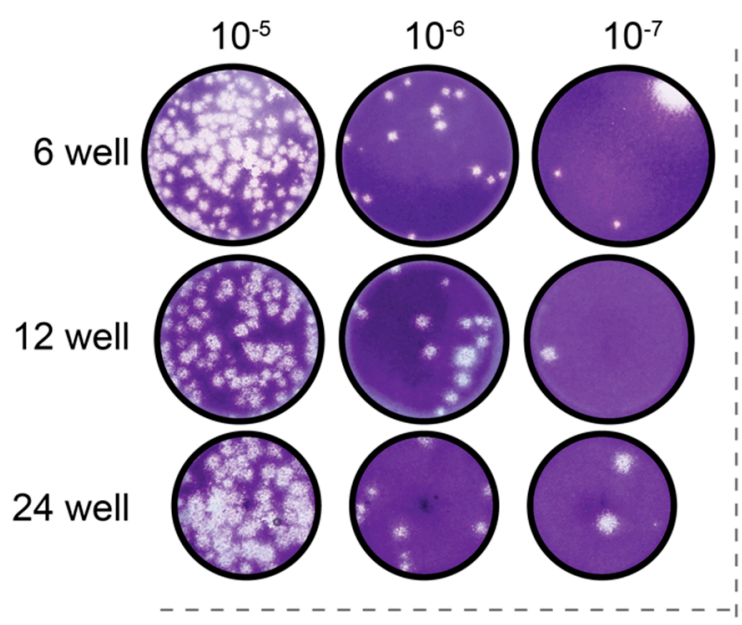

$0.6 \%$ Microcrystaline Cellulose
B.

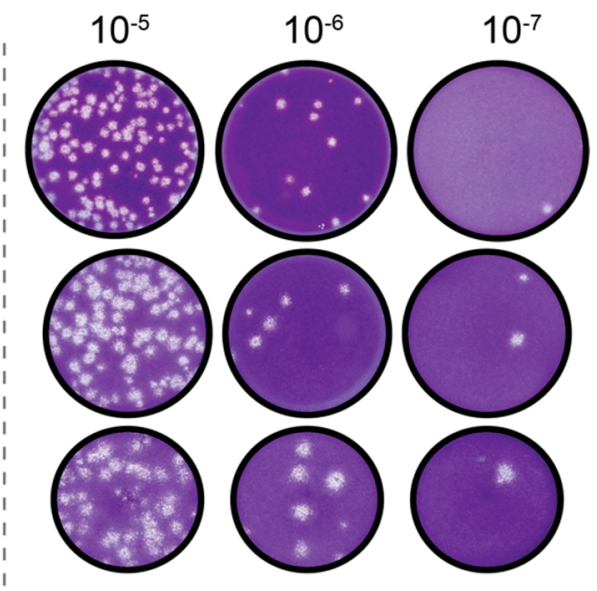

$1.2 \%$ Microcrystaline Cellulose

Figure 2. MCC is a suitable alternative as an overlay medium for SARS-CoV-2 plaque assays. Vero E6 cells were infected with serially diluted SARS-CoV-2 stock virus and overlaid with $0.6 \%$ (A) or $1.2 \%$ (B) MCC in serum free DMEM. After $72 \mathrm{~h}$, the MCC overlay was removed and monolayer were fixed and stained with crystal violet to visualize plaques.

\subsection{Development of a Foci Forming Assay for SARS-CoV-2}

For viruses like SARS-CoV-2 which produce significant CPE in permissive cell lines, traditional plaque assays are the standard for virus quantification. However, traditional plaque assays require waiting for a particular virus to produce significant enough $\mathrm{CPE}$ for quantifiable plaque formation. As described above, SARS-CoV-2 is most readily quantifiable by plaque assay 
at 3 days post-infection. Here, we set out to develop and immunohistochemical assay to reliably determine SARS-Cov-2 titers using a 96-well plate-based foci forming assay. Our results indicate that after only $24 \mathrm{~h}$ post infection, SARS-CoV-2 foci are readily detectable and quantifiable (Figure 3A). Although the titers obtained by the foci forming assay after $24 \mathrm{~h}$ incubation are approximately 1 log lower than titers obtained from traditional plaque assays at $72 \mathrm{~h}$, both data sets share similar trends (Figure 3B). Taken together, these data demonstrate that SARS-CoV-2 containing samples can be accurately quantified within $24 \mathrm{~h}$, and in a higher throughput manner than traditional plaque assays.

A.

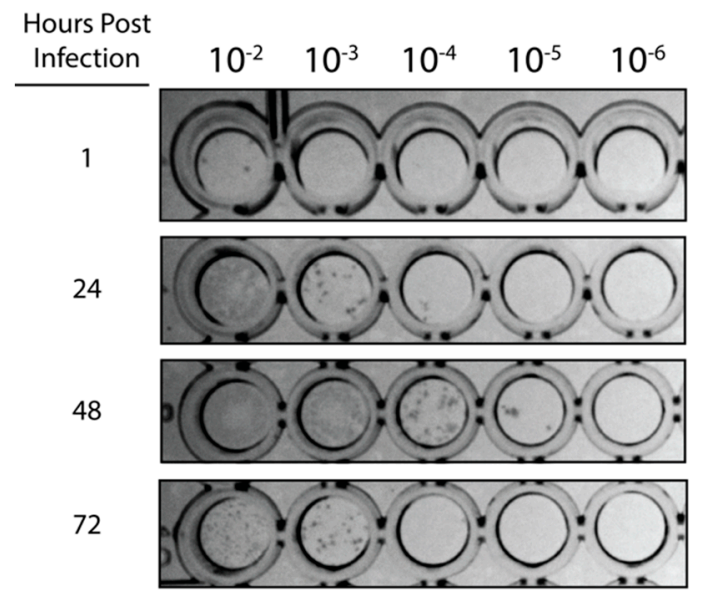

B.

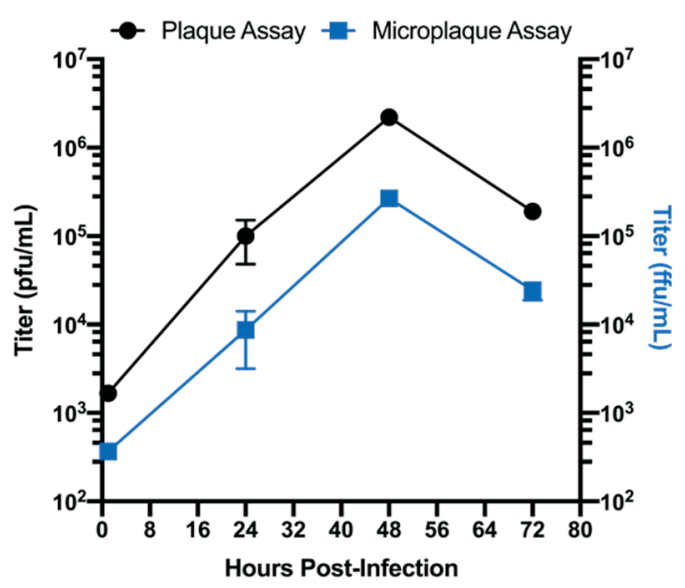

Figure 3. SARS-CoV-2 focus forming assay. (A) Representative image of the foci observed from serially diluted supernatants from infected Calu-3 cells. (B) Comparison of titers obtained from traditional plaque assay and focus forming assays on matched supernatants collected from SARS-CoV-2 infected Calu-3 cells. Data are representative of the mean and SEM of 3 replicates.

\subsection{Validation of Inactivation of SARS-CoV-2 by TRIzol, Formalin, Beta-Propiolactone, and Direct Heat}

Currently, there is limited data published on methods that successfully inactivate SARS-CoV-2. To help fill this gap in knowledge, we tested the ability of TRIzol, formalin, beta-propiolactone, and direct heat to successfully inactivate SARS-CoV-2.

\subsubsection{TRIzol}

TRIzol is a well-known and widely used reagent for the isolation from cells of nucleic acids and in some cases protein. TRIzol treated samples are generally very stable, and the ability to isolate RNA/DNA/protein without the need for column-based extraction kits with proprietary task-specific lysis buffers makes it an attractive option for processing SARS-CoV-2 infected samples. We determined that addition to SARS-CoV-2 stock virus $\left(1 \times 10^{6} \mathrm{pfu}\right.$; see Materials and Methods $)$ of TRIzol to a final concentration of $10 \%$ resulted in the successful inactivation of SARS-CoV-2 as treated samples resulted in no detectable cytopathic effect on Vero E6 cells $72 \mathrm{~h}$ post-infection (Table 1). 
Table 1. Inactivation of SARS-CoV-2 by TRIzol ${ }^{\circledR}$, formaldehyde, and beta-propiolactone.

\begin{tabular}{ccccc}
\hline & Treatment & \multicolumn{2}{c}{ CPE (72 Hours Post-Infection) } \\
\hline Trizol $^{\circledR}$ & Input (pfu) & Replicate 1 & Replicate 2 & Replicate 3 \\
\hline $10 \%$ & $1 \times 10^{6}$ & ND & ND & ND \\
\hline Formaldehyde & & & & \\
\hline $2 \%$ & Infected Cells (MOI 0.1) & ND & ND & ND \\
$1 \%$ & Infected Cells (MOI 0.1) & ND & ND & ND \\
$0.5 \%$ & Infected Cells (MOI 0.1) & ND & ND & ND \\
$0.1 \%$ & Infected Cells (MOI 0.1) & CPE & ND & ND \\
$0.05 \%$ & Infected Cells (MOI 0.1) & CPE & CPE & CPE \\
\hline Beta-propiolactone & & & & \\
\hline $0.5 \%$ & $1 \times 10^{6}$ & CD & CD & CD \\
$0.1 \%$ & $1 \times 10^{6}$ & CD & ND & ND \\
$0.05 \%$ & $1 \times 10^{6}$ & ND & ND & ND \\
\hline
\end{tabular}

CPE present (CPE), CPE not detected (ND), Cell death (CD).

\subsubsection{Formalin}

$10 \%$ neutral buffered formalin is a commonly used fixative for processing infected cells for downstream analysis such as microscopy. In order to determine the effectiveness of formaldehyde in inactivating SARS-CoV-2, Vero E6 cells were infected at an MOI of 0.01 and incubated at $37^{\circ} \mathrm{C}$ for $24 \mathrm{~h}$. After incubation, media was removed, and cells were treated with varying concentrations of formaldehyde for $1 \mathrm{~h}$ at room temperature. Fixed cells were then scraped off the dish and transferred to freshly plated Vero E6 cells. Our results indicate that formalin is effective in inactivating SARS-CoV-2 at concentrations ranging from $0.5 \%$ to $2 \%$ (total formaldehyde concentration) after $1 \mathrm{~h}$ at room temperature (Table 1). While the exact viral load inactivated was not determined, the infections for this study were performed in a manner analogous to those shown in Figure 1C, where viral titers were high at $24 \mathrm{~h}$ post-infection (Figure 1C). Given that $10 \%$ neutral buffered formalin (NBF) contains $4 \%$ formaldehyde, treatment of SARS-CoV-2 containing samples with $10 \% \mathrm{NBF}$ for $1 \mathrm{~h}$ at room temperature is more than adequate for SARS-CoV-2 inactivation. Our data also indicates that lower concentrations (down to $0.5 \%$ total formaldehyde concentration) will effectively inactivate SARS-CoV-2 for the purposes that involve the processing liquid samples, such as the isolation of whole virions. Therefore, fixative solutions containing a final concentration of formaldehyde above $0.5 \%$ should effectively inactivate SARS-CoV-2. Additionally, as described in Figure 3, SARS-CoV-2 N protein could be readily detected in fixed infected Vero E6 monolayers, suggesting that treatment with 10\% neutral-buffered formalin for $1 \mathrm{~h}$ at room temperature is compatible with viral antigen detection.

\subsubsection{Beta-Propiolactone}

Beta-propiolactone (BPL) is a commonly used reagent for the inactivation of viruses for use in vaccine preparations [11-14] and it has recently been used in the development of an inactivated SARS-CoV-2 vaccine preparation [15]. Our results indicate that incubation of SARS-CoV-2 $\left(1 \times 10^{6} \mathrm{pfu}\right)$ in solution with $0.5 \%$ BPL for $16 \mathrm{~h}$ at $4 \mathrm{C}$ followed by a 2-h incubation at $37^{\circ} \mathrm{C}$ results in complete inactivation of infectious SARS-CoV-2 (Table 1). Examination of control cells treated with $0.5 \%$ BPL by light microscopy and crystal violet staining indicated no apparent cytotoxicity $72 \mathrm{~h}$ post-treatment.

Given its use in the preparation of vaccines, we wanted to determine if BPL could provide a rapid method for the purification of inactivated viral particles. Our results indicate that after purification of BPL treated SARS-CoV-2 stocks over a $20 \%$ sucrose gradient (Figure $4 \mathrm{~A}$ ) intact viral particles are readily apparent by electron microscopy (Figure 4B). We also demonstrated that both nucleoprotein and spike (full-length and cleaved) of SARS-CoV-2 are detectable by western blot in these samples (Figure 4C). These results indicate that BPL inactivation of SARS-CoV-2 viral particles and their subsequent purification will yield inactivated, intact viral particles. 
A.

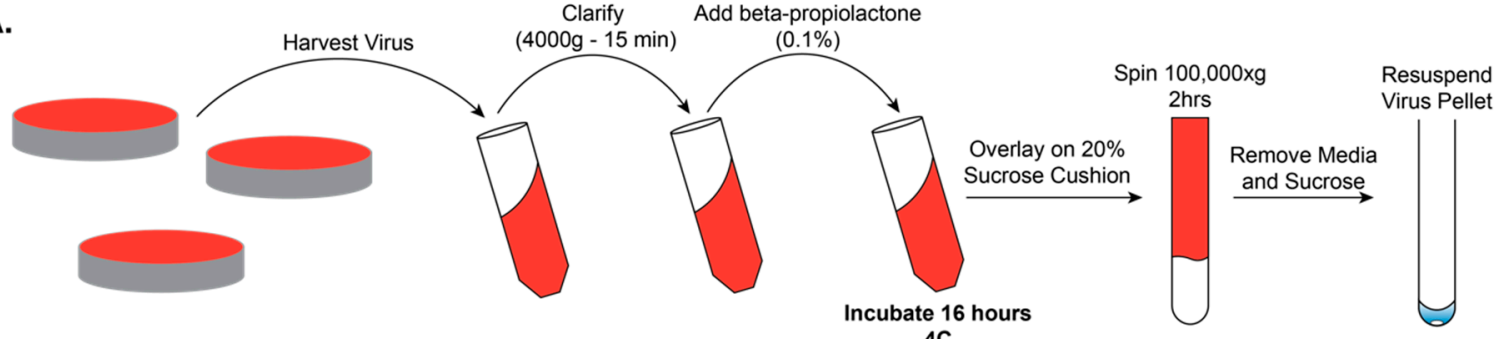

B.

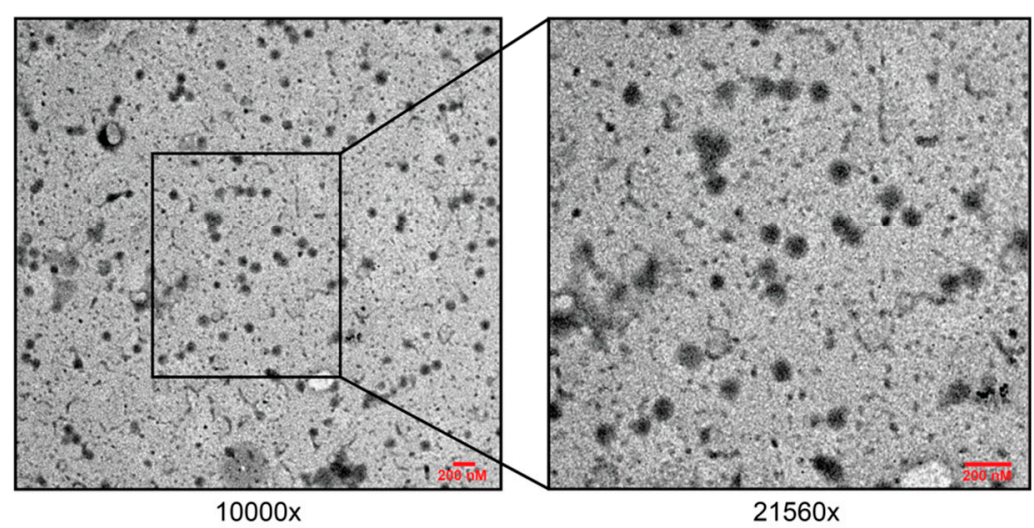

c.

.

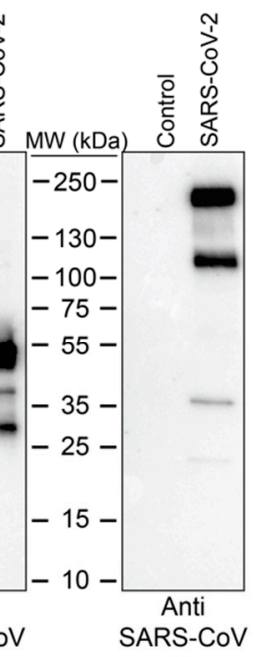

Figure 4. BPL inactivation and purification of SARS-CoV-2 virus particles. (A) Graphical depiction of the workflow established for the inactivation and purification of BPL inactivated SARS-CoV-2. (B) Negative stain transmission electron microscope (TEM) images of SARS-CoV-2 virus particles after purification from cell culture media over a $20 \%$ sucrose cushion at $100,000 \times g$ for $2 \mathrm{~h}$. Scale bars represent $200 \mathrm{nM}$. (C) Western blots of BPL inactivated virus particles for SARS-CoV-2 nucleoprotein and spike protein.

\subsubsection{Heat}

To determine the effectiveness of heat in inactivating SARS-CoV-2 with respect to time, we heated virus stocks at $100{ }^{\circ} \mathrm{C}$ for 5,10 , and $15 \mathrm{~min}$ and $56{ }^{\circ} \mathrm{C}$ for $15,30,45$, and $60 \mathrm{~min}$. After heating, the remaining infectious SARS-CoV-2 was quantified by plaque assay. Treatment of SARS-CoV-2 $\left(1 \times 10^{6} \mathrm{pfu}\right)$ for $5 \mathrm{~min}$ at $100{ }^{\circ} \mathrm{C}$ and $45 \mathrm{~min}$ at $56^{\circ} \mathrm{C}$ resulted in complete inactivation of infectious virus (Figure 5). 


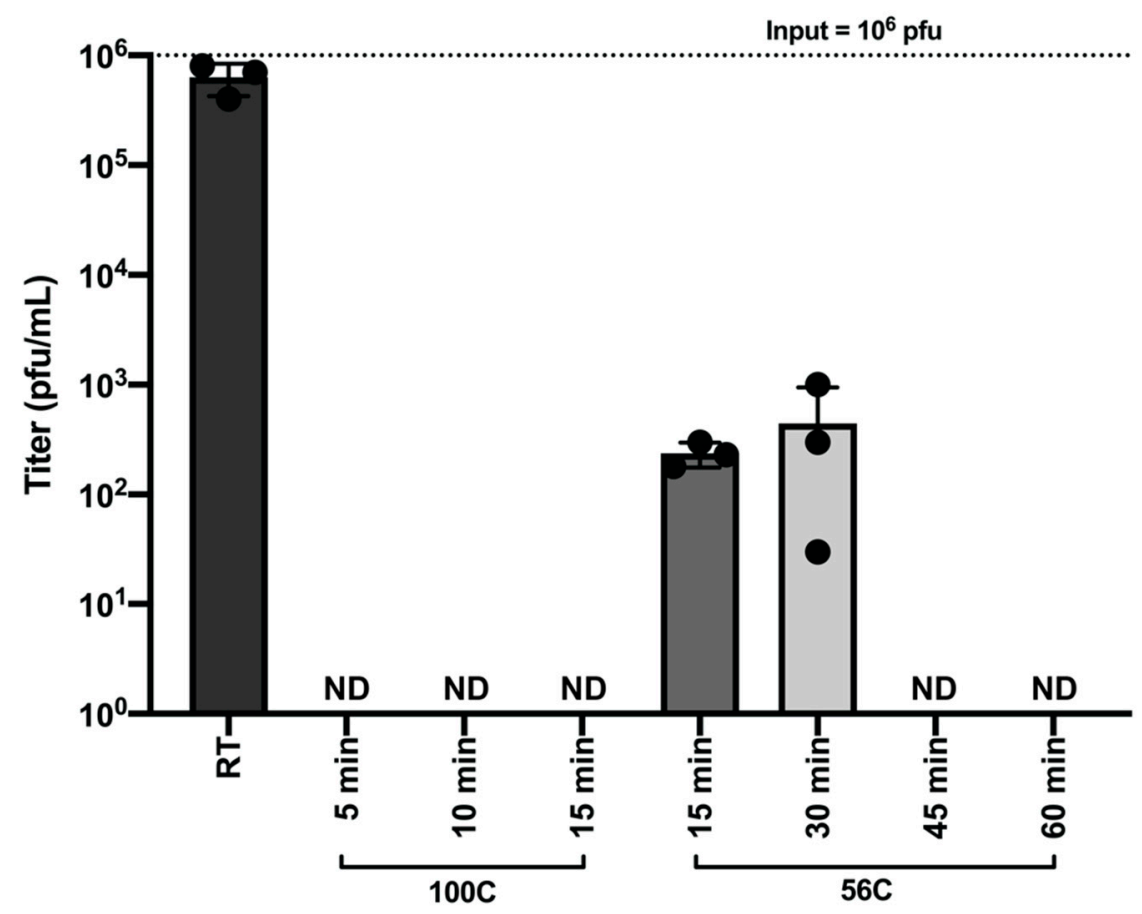

Figure 5. Heat Inactivation of SARS-CoV-2. SARS-CoV-2 containing samples $\left(1 \times 10^{6} \mathrm{pfu}\right)$ were heated at $100{ }^{\circ} \mathrm{C}$ for 5,10 and $15 \mathrm{~min}$ and $56^{\circ} \mathrm{C}$ for $15,30,45$, and $60 \mathrm{~min}$. Samples were assayed by plaque assay to detect remaining infectious virus post-heating. The room temperature control was incubated at room temperature until all heated samples were prepared. Data are representative of the mean and SEM of 3 replicates.

\section{Discussion}

The ability to grow and accurately quantify infectious virus is critical for virological studies. Here, we sought to determine the growth kinetics of SARS-CoV-2 in several commonly used cell lines as well as the most appropriate time post-infection to accurately quantify SARS-CoV-2 by plaque assay. Significant growth was achieved in both Vero E6 and Calu-3 cells at the time points tested. We also observed that the colorectal adenocarcinoma Caco-2 cell line was able to propagate SARS-CoV-2, albeit to lower titers than Vero E6 and Calu-3 cells. Based on the replication kinetics observed in Caco-2 cells, it is likely that if the infection were allowed to progress past $72 \mathrm{~h}$ that higher titers would have been achieved. Huh7 and 293T cells exhibited modest growth of SARS-CoV-2, and A549 cells did not support SARS-CoV-2 replication at any of the tested timepoints. Therefore, these cell lines are not ideal hosts for virological studies with SARS-CoV-2 in the absence of modifications such as overexpression of the SARS-CoV-2 entry receptor ACE2 or the cellular protease TMPRSS2 which is necessary for Spike processing [8,9].

Plaque assays are among the most commonly used techniques for the accurate quantification of infectious virus. To establish reliable plaque assays for SARS-CoV-2 we compared two separate approaches. One used an agarose overlay and the other used microcrystalline cellulose (MCC). In our hands, both approaches yielded comparable data. However, the MCC overlay approach has significant advantages over the more traditional agarose overlay. MCC overlays do not require heating prior to use which significantly expedites the processing of large numbers of plaque assays and also alleviates concerns of overheating cell monolayers. MCC overlays are also insensitive to virus inoculum remaining in place providing the researcher with the option to remove virus inoculum or not. Additionally, MCC overlays do not require the removal of a "plug" like in the use of agarose overlays as MCC overlays remain liquid throughout their use. They can simply be aspirated, and the monolayer can be further processed. However, due to the fact that MCC overlays remain liquid throughout the 
course of the assay, it is important to take care that plaque assays utilizing MCC as an overlay are not moved until harvested to ensure reliable data. Our data presented here demonstrate that MCC is an effective and efficient overlay alternative to agarose for SARS-CoV-2 plaque assays.

While plaque assays are perhaps the most commonly used technique for the quantification of infectious virus, immunohistochemical focus forming assays are also commonly used for the quantification of virus [10,16-18]. We have established that SARS-CoV-2 can be reliably quantified by a 96-well plate-based focus forming assay in only $24 \mathrm{~h}$. When compared to traditional plaque assays for SARS-CoV-2 which require $72 \mathrm{~h}$ for easily quantifiable plaques, the ability to quantify infectious SARS-CoV-2 within $24 \mathrm{~h}$ in a 96-well plate format represents a significant advantage for studies requiring higher-throughput. While it is important to acknowledge that titers acquired with a focus forming assay will generally be one log lower than those acquired by traditional plaque assay, the focus forming assay described here is fully capable of differentiating log-scale changes in virus titer. Taken together, the focus forming assay described here is a rapid and efficient method for quantifying infectious SARS-CoV-2.

Currently the recommendation from the Centers for Disease Control and Prevention (CDC) is for studies involving the propagation of SARS-CoV-2 to be performed within BSL3 laboratories with standard BSL3 practices. While an official risk group determination has not been made for SARS-CoV-2, the related viruses SARS-CoV and MERS are classified as risk-group 3 pathogens. As a BSL3 pathogen, having validated methods to inactivate SARS-CoV-2 is important so as to ensure safety while also allowing removal of samples to lower biosafety levels for analysis. For example, whole inactivated virus can serve as antigen for immunological assays, vaccine preparations, and for the analysis of virus composition. We chose to examine beta-propiolactone as a means to inactivate SARS-CoV-2. This method was chosen because it preserves virus structure and antigenicity, and it has recently been used to generate an inactivated vaccine preparation for SARS-CoV-2 [15]. Our data show that treatment of SARS-CoV-2 with beta-propiolactone at a concentration of $0.5 \%$ for $16 \mathrm{~h}$ at $4{ }^{\circ} \mathrm{C}$ followed by $2 \mathrm{~h}$ at $37^{\circ} \mathrm{C}$ will yield intact viral particles that can be utilized safely for downstream purposes.

The isolation of viral RNA and protein from virus or virus infected cells or the isolation RNA from host cells is critical to characterize virus sequence variation and to study the impact of infection on host gene expression. TRIzol is a commonly used reagent for RNA isolation that can also be used to for DNA and protein isolation. Our data show that treatment of SARS-CoV-2 containing samples with TRIzol following the manufacturer's instructions is an effective method of inactivating SARS-CoV-2.

Formaldehyde is a ubiquitously used reagent in life sciences and has numerous applications such as the fixation of cells for microscopy studies such as indirect immunofluorescence of infected cells and as a disinfectant for scientific equipment. Here, we demonstrate that treatment of SARS-CoV-2 infected cells with formaldehyde at a concentration at or above $0.5 \%$ for one hour at room temperature effectively inactivates SARs-CoV-2. Given the wide ranges of uses for formaldehyde, we hope this data will assist in the safe processing of probable or confirmed SARS-CoV-2 containing samples.

Analyses of host responses to viral infection either through the use of Western blotting or the determination of virus specific antibodies in patient sera generally require heating of the sample prior to downstream analysis. To this end, we have determined that heating of SARS-CoV-2 containing samples at $100{ }^{\circ} \mathrm{C}$ for longer than $5 \mathrm{~min}$ is a safe and effective method for the downstream analysis of samples by Western blot. Likewise, our data demonstrate that heat treatment of laboratory or clinical samples at $56{ }^{\circ} \mathrm{C}$ for one hour will serve to effectively inactivate SARS-CoV-2. However, it has been recently reported that heat treatment of clinical samples such as serum can negatively impact sample quality for downstream processes, so researchers utilizing heat treatment as a method of inactivation should take this into account in their experimental design [19].

Taken together, we hope the methods and data reported here will serve to expedite the much-needed research required to address this unprecedented pandemic. 
Author Contributions: A.S.J., J.A.S., and C.F.B. conceptualized experiments. A.S.J. and J.A.S. performed experiments and analyzed data. A.S.J., J.A.S., and C.F.B. wrote the manuscript. All authors have read and agreed to the published version of the manuscript.

Funding: This research was funded by NIH grants to C.F.B., including P01AI120943 and R01AI143292.

Acknowledgments: We would like to thank Natasha K. Griffith, Marty Wildes, and Michael Walsh of the GSU High Containment Core for their support in completing the studies reported here.

Conflicts of Interest: The authors declare no conflict of interest.

\section{References}

1. Bedford, J.; Enria, D.; Giesecke, J.; Heymann, D.L.; Ihekweazu, C.; Kobinger, G.; Lane, H.C.; Memish, Z.; Oh, M.D.; Sall, A.A.; et al. COVID-19: Towards controlling of a pandemic. Lancet 2020, 395, 1015-1018. [CrossRef]

2. World Health Organization. Situation Report-112 Coronavirus Disease 2019 (COVID-19); World Health Organization: Geneva, Switzerland, 2020.

3. Blow, J.A.; Dohm, D.J.; Negley, D.L.; Mores, C.N. Virus inactivation by nucleic acid extraction reagents. J. Virol. Methods 2004, 119, 195-198. [CrossRef] [PubMed]

4. Darnell, M.E.; Subbarao, K.; Feinstone, S.M.; Taylor, D.R. Inactivation of the coronavirus that induces severe acute respiratory syndrome, SARS-CoV. J. Virol. Methods 2004, 121, 85-91. [CrossRef] [PubMed]

5. Haddock, E.; Feldmann, F.; Feldmann, H. Effective Chemical Inactivation of Ebola Virus. Emerg. Infect. Dis. 2016, 22, 1292-1294. [CrossRef] [PubMed]

6. Rabenau, H.F.; Cinatl, J.; Morgenstern, B.; Bauer, G.; Preiser, W.; Doerr, H.W. Stability and inactivation of SARS coronavirus. Med. Microbiol. Immunol. 2005, 194, 1-6. [CrossRef] [PubMed]

7. Lei, S.; Gao, X.; Sun, Y.; Yu, X.; Zhao, L. Gas chromatography-mass spectrometry method for determination of beta-propiolactone in human inactivated rabies vaccine and its hydrolysis analysis. J. Pharm. Anal. 2018, 8, 373-377. [CrossRef] [PubMed]

8. Hoffmann, M.; Kleine-Weber, H.; Schroeder, S.; Kruger, N.; Herrler, T.; Erichsen, S.; Schiergens, T.S.; Herrler, G.; Wu, N.H.; Nitsche, A.; et al. SARS-CoV-2 Cell Entry Depends on ACE2 and TMPRSS2 and Is Blocked by a Clinically Proven Protease Inhibitor. Cell 2020, 181, 271-280. [CrossRef] [PubMed]

9. Matsuyama, S.; Nao, N.; Shirato, K.; Kawase, M.; Saito, S.; Takayama, I.; Nagata, N.; Sekizuka, T.; Katoh, H.; Kato, F.; et al. Enhanced isolation of SARS-CoV-2 by TMPRSS2-expressing cells. Proc. Natl. Acad. Sci. USA 2020, 117, 7001-7003. [CrossRef] [PubMed]

10. Matrosovich, M.; Matrosovich, T.; Garten, W.; Klenk, H.D. New low-viscosity overlay medium for viral plaque assays. Virol. J. 2006, 3, 63. [CrossRef] [PubMed]

11. Budowsky, E.I.; Friedman, E.A.; Zheleznova, N.V.; Noskov, F.S. Principles of selective inactivation of viral genome. VI. Inactivation of the infectivity of the influenza virus by the action of beta-propiolactone. Vaccine 1991, 9, 398-402. [CrossRef]

12. Budowsky, E.I.; Smirnov Yu, A.; Shenderovich, S.F. Principles of selective inactivation of viral genome. VIII. The influence of beta-propiolactone on immunogenic and protective activities of influenza virus. Vaccine 1993, 11, 343-348. [CrossRef]

13. Chowdhury, P.; Topno, R.; Khan, S.A.; Mahanta, J. Comparison of beta-Propiolactone and Formalin Inactivation on Antigenicity and Immune Response of West Nile Virus. Adv. Virol. 2015, 2015, 616898. [CrossRef] [PubMed]

14. Logrippo, G.A.; Hartman, F.W. Antigenicity of beta-propiolactone-inactivated virus vaccines. J. Immunol. 1955, 75, 123-128. [PubMed]

15. Gao, Q.; Bao, L.; Mao, H.; Wang, L.; Xu, K.; Yang, M.; Li, Y.; Zhu, L.; Wang, N.; Lv, Z.; et al. Rapid development of an inactivated vaccine candidate for SARS-CoV-2. Science 2020. [CrossRef] [PubMed]

16. Cruz, D.J.; Shin, H.J. Application of a focus formation assay for detection and titration of porcine epidemic diarrhea virus. J. Virol. Methods 2007, 145, 56-61. [CrossRef] [PubMed]

17. Muller, C.; Hardt, M.; Schwudke, D.; Neuman, B.W.; Pleschka, S.; Ziebuhr, J. Inhibition of Cytosolic Phospholipase A2alpha Impairs an Early Step of Coronavirus Replication in Cell Culture. J. Virol. 2018, 92. [CrossRef] 
18. Ye, C.; Wang, D.; Liu, H.; Ma, H.; Dong, Y.; Yao, M.; Wang, Y.; Zhang, H.; Zhang, L.; Cheng, L.; et al. An Improved Enzyme-Linked Focus Formation Assay Revealed Baloxavir Acid as a Potential Antiviral Therapeutic Against Hantavirus Infection. Front. Pharm. 2019, 10, 1203. [CrossRef] [PubMed]

19. Hu, X.; An, T.; Situ, B.; Hu, Y.; Ou, Z.; Li, Q.; He, X.; Zhang, Y.; Tian, P.; Sun, D.; et al. Heat inactivation of serum interferes with the immunoanalysis of antibodies to SARS-CoV-2. medRxiv 2020. [CrossRef]

(C) 2020 by the authors. Licensee MDPI, Basel, Switzerland. This article is an open access article distributed under the terms and conditions of the Creative Commons Attribution (CC BY) license (http://creativecommons.org/licenses/by/4.0/). 